\title{
Combination of Advanced Lung Cancer Inflammation Index and C-Reactive Protein Is a Prognostic Factor in Patients With Operable Non-Small Cell Lung Cancer
}

\author{
Masaki Tomita $^{\mathrm{a}, \mathrm{c}}$, Takanori Ayabe ${ }^{\mathrm{a}}$, Ryo Maeda ${ }^{\mathrm{a}}$, Kunihide Nakamura ${ }^{\mathrm{b}}$
}

\begin{abstract}
Background: Previous study of ours showed that advanced lung cancer inflammation index (ALI) and C-reactive protein (CRP) are independent significant prognostic factors in operable non-small cell lung cancer (NSCLC) patients. Since both ALI and CRP are markers of inflammation, the aim of this study was to examine whether the combination of $\mathrm{ALI}$ and CRP is a prognostic indicator of resected NSCLC or not.
\end{abstract}

Methods: Three hundred forty-one NSCLC patients who underwent surgery at our institution between 2008 and 2012 were included. ALI score was calculated as BMI $\times$ ALB/NLR with BMI indicating body mass index, ALB indicating serum albumin and NLR indicating neutrophil lymphocyte ratio. The prognostic significance of the combination of ALI and CRP was evaluated.

Results: Compared to patients with high ALI (> 37.67) group, low ALI group had significantly poorer cancer-specific survival. Similarly, patients with high CRP ( $>0.15 \mathrm{mg} / \mathrm{dL})$ had poorer survival. Based on the combination of ALI and CRP, patients with high ALI and low CRP were assigned an ALI_CRP score of 0, those with both high or both low were assigned an ALI_CRP score of 1, and those with low ALI and high CRP were assigned an ALI CRP score of 2. The patients' cancer-specific survival was significantly related to this ALI_CRP score. On multivariate analysis, gender, histology, pN status, serum carcinoembryonic antigen (CEA) level and the ALI_CRP score were associated independently with cancer-specific survival.

Conclusions: The combination of ALI and CRP is a useful predictor of overall survival and could be a simple prognostic tool to help identify operable NSCLC patients.

Keywords: Advanced lung cancer inflammation index; C-reactive

Manuscript submitted December 12, 2017, accepted December 20, 2017

aDepartment of Thoracic and Breast Surgery, Faculty of Medicine, University of Miyazaki, Kihara 5200, Kiyotake, Miyazaki 889-1692, Japan

bDepartment of Cardiovascular Surgery, Faculty of Medicine, University of Miyazaki, Kihara 5200, Kiyotake, Miyazaki 889-1692, Japan

${ }^{\mathrm{c} C}$ Corresponding Author: Masaki Tomita, Department of Thoracic and Breast Surgery, Faculty of Medicine, University of Miyazaki, Kihara 5200, Kiyotake, Miyazaki 889-1692, Japan. Email: mtomita@med.miyazaki-u.ac.jp

doi: https://doi.org/10.14740/wjon1076w protein; Non-small cell lung cancer; Surgery; Prognosis

\section{Introduction}

Over the past years, it has been well accepted that the host inflammatory response has an important role in the development and progression of cancer, and several previous studies reported that systemic inflammation is useful for predicting the survival rates of cancer patients $[1,2]$. Emerging evidence indicates that preoperative markers of inflammation have been reported to be significant factors for prognosis of surgery patients [3].

With regard to operable non-small cell lung cancer (NSCLC), the presence of a systemic inflammation response as evidenced by an elevated C-reactive protein (CRP) concentration, is associated with poor survival in patients with NSCLC $[4,5]$. Moreover, several studies have shown that inflammation-based prognostic scores including the neutrophil to lymphocyte ratio (NLR) and a combination of serum CRP and albumin as the Glasgow prognostic score (GPS) are associated with survival in patients with operable NSCLC [6-8].

Furthermore, Jafri et al [9] evaluated a novel inflammationbased prognostic system, named advanced lung cancer inflammation index (ALI; based on body mass index (BMI), serum albumin (ALB) and NLR) in patients with metastatic NSCLC. Recently, we reported a prognostic significance of ALI in operable NSCLC [10]. Both ALI and CRP are markers of inflammation. Our previous results showed that both ALI and CRP were independent prognostic factors in multivariable analysis [11]. From this result, the underlying prognostic mechanism of ALI and CRP might be different. Therefore, we considered that the combination of ALI and CRP might be a more useful prognostic inflammation marker for operable NSCLC.

\section{Patients and Methods}

This retrospective study was approved by our institutional review board approval, and the need to obtain patient consent was waived. We retrospectively reviewed data from 341 patients (173 men and 168 women, with a median age of 69 years old) with resectable NSCLC who received surgery at 

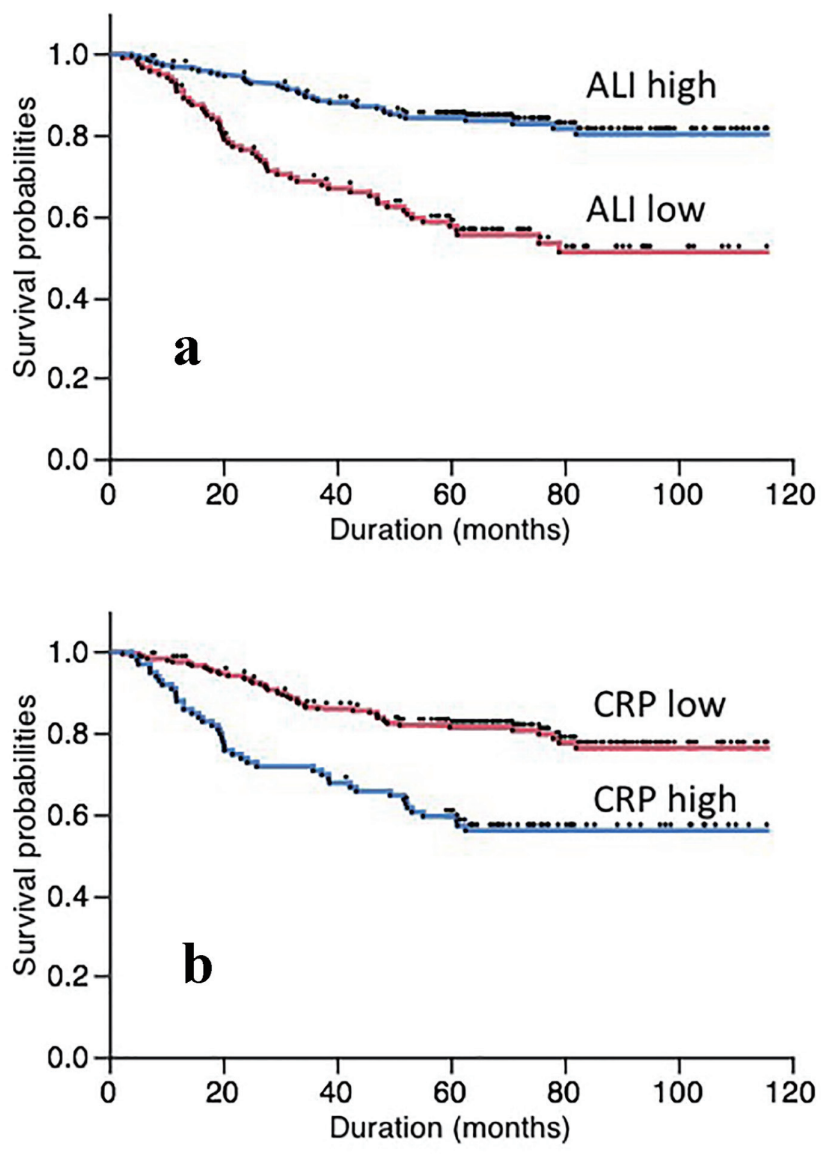

Figure 1. Overall postoperative cancer specific survival of patients based on ALI (a) and CRP (b).

our hospital between 2008 and 2012. The criteria for inclusion in this study included: complete pulmonary resection and systematic node dissection of the hilar and mediastinal lymph nodes, histologically confirmed NSCLC and complete clinical, laboratory, imaging and follow-up data. The exclusion criteria included: preoperative chemotherapy/radiotherapy or died perioperative period, clinical evidence of infection or other bone marrow, hematological or autoimmune disease, a history of lung cancer, or a second primary cancer diagnosed within 5 years of the lung cancer index. The median postoperative follow-up was 62.5 months. The blood samples for these analyses were collected before surgery. The serum levels of CRP were measured by a latex photometric immunoassay (Mitsubishi Kagaku Iatron, Tokyo, Japan). The cut-off value of CRP was set as $0.15 \mathrm{mg} / \mathrm{dL}$ according to the manufacturer's instructions. The preoperative serum carcinoembryonic antigen (CEA) and cytokeratin 19 fragments (CYFRA 21-1) levels were also measured in all patients by enzyme immunoassay in a single laboratory at our hospital. The preoperative BMI was calculated as weight in kilograms divided by height in meters squared. ALI was calculated as follows: ALI $=\mathrm{BMI} \times \mathrm{ALB}(\mathrm{g} /$ $\mathrm{dL}$ )/NLR [9]. The optimal cut-off value of ALI for overall survival was determined by Cutoff Finder (http://molpath.charite. de/cutoff) [12].
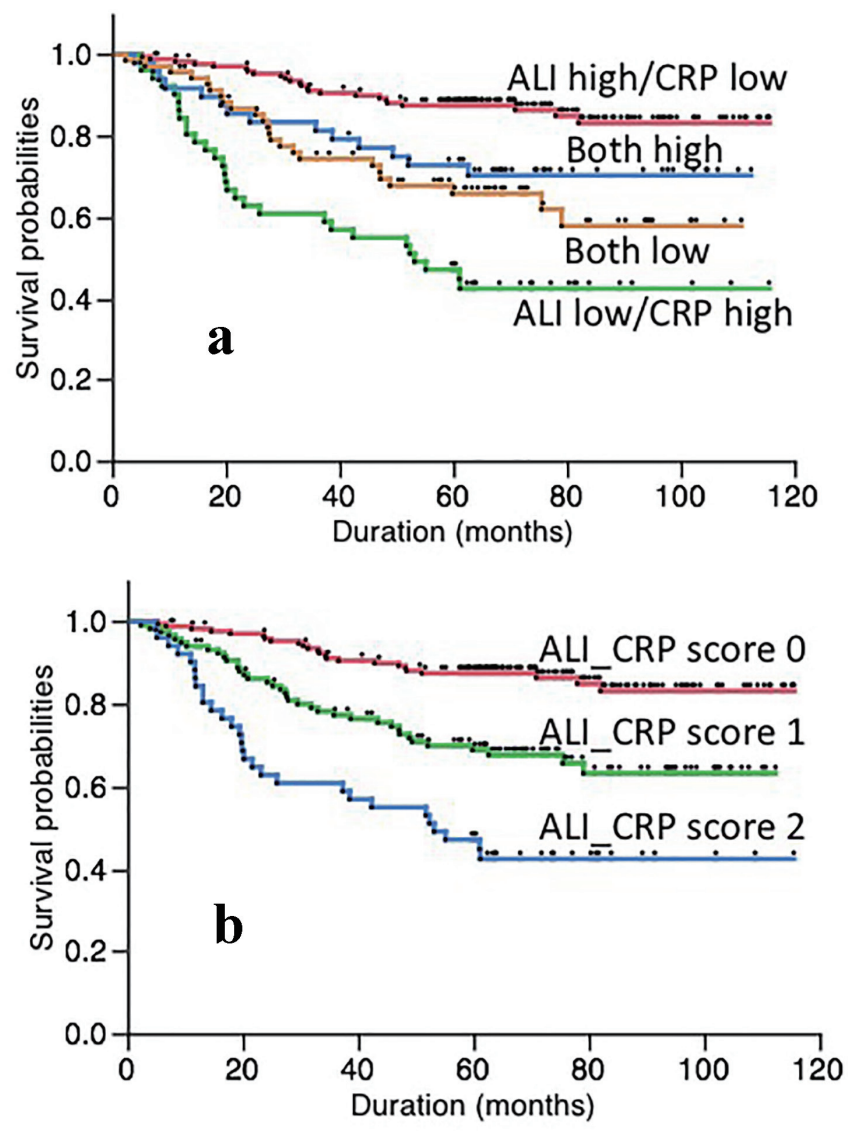

Figure 2. Overall postoperative cancer specific survival of patients based on the combination of ALI and CRP (a) and the ALI_CRP score (b).

Comparison of data between the groups was made by using Fisher's extract test. Cumulative cancer-specific survival curves after surgery were calculated using the Kaplan-Meier method and differences were evaluated using the log-rank test. The Cox proportional hazard model was performed in univariable and multivariable analyses, and hazard ratios estimated from the Cox analysis are reported as relative risks with corresponding 95\% confidence intervals. Statistical analyses were performed using JMP (SAS Institute Inc., Cary, NC, USA). A P $<0.05$ was considered to indicate statistical significance.

\section{Results}

We used Cutoff Finder [12] to determine a cut-off point, and the optimal cut-off point of ALI was determined to be 37.67. Based on this cut-off value, patients were subdivided into low ALI $($ ALI < 37.67) and high ALI (ALI > 37.67) groups. There are 120 patients $(35.28 \%)$ in low ALI group; the remaining 221 patients $(64.72 \%)$ are in high ALI group.

Comparison of overall postoperative cancer-specific survival curves between the low and high ALI groups was shown in Figure 1a. Overall postoperative cancer-specific survival was significantly higher in the high ALI group than in the low 
Table 1. Clinical Characteristics of Patients Based on the ALI_CRP Score

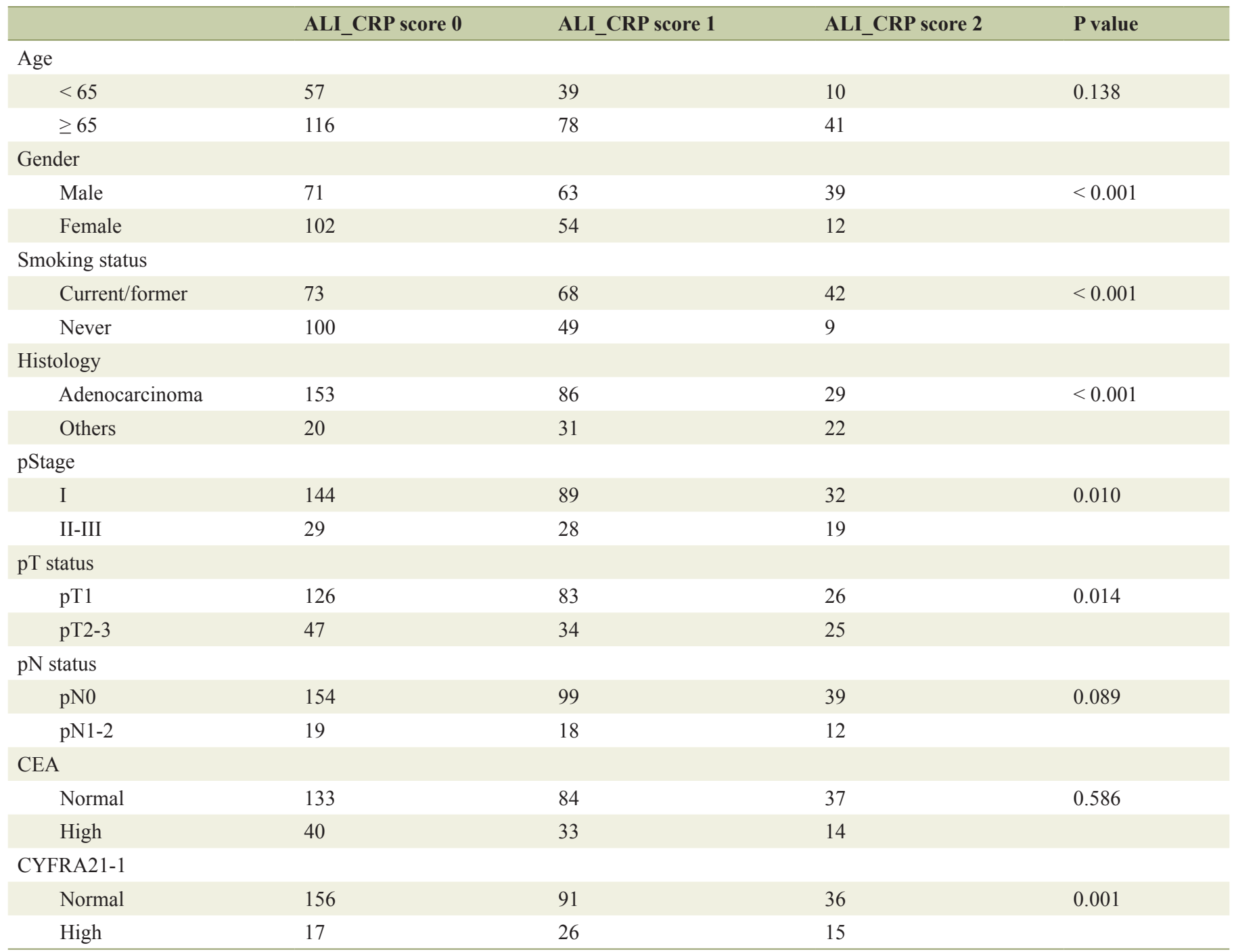

ALI: advanced lung cancer inflammation index; CRP: C-reactive protein; CEA: carcinoembryonic antigen; CYFRA21-1: cytokeratin 19 fragments.

ALI group ( $\mathrm{P}<0.001)$. Similarly, comparison of postoperative cancer-specific survival curves according to CRP showed that there was a significant difference for patients' survival (Fig. 1b). The patients with high CRP $(>0.15 \mathrm{mg} / \mathrm{dL})$ had a significantly worse survival than those with low CRP $(\mathrm{P}<0.001)$.

The overall postoperative cancer-specific survival was also examined using the combination of ALI and CRP. Fiveyear overall cancer-specific survival was significantly higher in the patients with high ALI and low CRP than in those with low ALI and high CRP (87.44\% vs. 47.06\%; P < 0.001). The survival rates of patients with both high or both low of ALI and CRP were intermediate values (Fig. 2a). Based on this result, patients were subdivided into three groups using the ALI_CRP score. Briefly, patients with both a low ALI $(<37.67)$ and high CRP $(>0.15 \mathrm{mg} / \mathrm{dL})$ were allocated a score of 2 (ALI_CRP score 2$)$. Accordingly, patients with a high ALI $(>37.6 \overline{7})$ and low CRP $(<0.15 \mathrm{mg} / \mathrm{dL})$ were allocated a score of 0 (ALI CRP score 0$)$. Remaining patients with both high or low of
ALI and CRP were allocated a score of 1 (ALI_CRP score 1).

The clinicopathological characteristics of the patients based on this ALI_CRP score are shown in Table 1. This ALI_ CRP score was related to gender, smoking status, histology, pStage, pT status, serum CYFRA21-1 level, but not related to age, $\mathrm{pN}$ status and serum CEA level. The survival curve based on this ALI_CRP score was shown in Figure 2b.

By univariate analysis, we found that the ALI_CRP score was correlated with overall postoperative cancer-specific survival $(\mathrm{P}<0.001)$ (Table 2$)$. Factors including male gender $(\mathrm{P}$ $<0.001)$, smoking status $(\mathrm{P}<0.001)$, histology $(\mathrm{P}<0.001)$, $\mathrm{pT}$ status $(\mathrm{P}<0.001)$, pN status $(\mathrm{P}<0.001)$, serum $\mathrm{CEA}$ level $(\mathrm{P}$ $<0.001)$, and serum CYFRA21-1 level $(\mathrm{P}<0.0001)$ were also significant for overall postoperative cancer-specific survival. These clinical characteristics that were found significant $(\mathrm{P}$ $<0.05$ ) by univariate analysis were assessed by a multivariate analysis. Our results revealed that the ALI_CRP score was correlated with overall postoperative cancer-specific survival, 
Table 2. Univariate Analysis

\begin{tabular}{|c|c|c|c|c|c|c|}
\hline & Foverable & Unfayerable & Horard ratio & P yolue & $95 \% \mathrm{cc}$ & nce interval \\
\hline & Favoravie & Uimavoravie & Hazar Tatio & I value & Lower & Upper \\
\hline Age & $<65$ & $\geq 65$ & 0.757 & 0.240 & 0.462 & 1.196 \\
\hline Smoking status & Never & Current/former & 0.349 & $<0.001$ & 0.215 & 0.548 \\
\hline Histology & Adenocarcinoma & Others & 0.249 & $<0.001$ & 0.164 & 0.380 \\
\hline CEA & Normal & High & 0.380 & $<0.001$ & 0.251 & 0.584 \\
\hline CYFRA21-1 & Normal & High & 0.352 & $<0.001$ & 0.229 & 0.555 \\
\hline ALI_CRP score & Score 0 & Others & 0.287 & $<0.001$ & 0.177 & 0.451 \\
\hline
\end{tabular}

ALI: advanced lung cancer inflammation index; CRP: C-reactive protein; CEA: carcinoembryonic antigen; CYFRA21-1: cytokeratin 19 fragments.

while smoking status, pT status and serum CYFRA21-1 level were not significant factors in predicting overall postoperative cancer-specific survival. Furthermore, gender, histology, pN status and serum CEA level were also revealed as independent prognostic factors (Table 3 ).

\section{Discussion}

It is well accepted that systemic inflammation appears to play a pivotal role in the progression of NSCLC by promoting tumor angiogenesis, tumor metastasis and cancer cell proliferation [1-3]. Elevated preoperative inflammatory markers have been known to be inversely related to survival outcomes [1-3]. The NLR, representing a combination of circulating neutrophil and lymphocyte counts, can reflect the imbalance between neutrophils and lymphocytes in patients with tumors and serves as a representative index of systemic inflammation $[7,8]$. Previous meta-analyses of published articles demonstrated that an elevated NLR was a predictor of poor overall survival in patients with NSCLC $[7,8]$.

Impaired nutrition has been well known as an important prognostic predictor after an operation for NSCLC [12]. In general, preoperative nutritional status was usually examined using ALB, BMI and a recent history of weight loss. Previous study reported that low BMI is a predictor of survival in the long term independently of tumor extension and staging in resected NSCLC [12]. Furthermore, ALB is also a good marker of nutritional status, and has been reported to be associated with survival in NSCLC surgery patients [13]. Taken together, BMI and ALB might serve as prognostic indicators. ALI was calculated by multiplying BMI by ALB/ NLR [9]. Since previous studies showed the prognostic significance of BMI, ALB and NLR $[7,8,12,13]$, it is easy to understand the reason for the usefulness of ALI. Our previous result showed that ALI was an independent prognostic factor [10].

CRP is also a sensitive marker of systemic inflammation $[4,5]$. Previous meta-analyses of published articles also demonstrated that an elevated CRP was a predictor of poor overall survival in patients with NSCLC $[4,5]$. Both NLR and CRP are markers of inflammation. However, in the previous result of ours, both NLR based ALI and CRP were independent prognostic factors in resected NSCLC patients [10]. From this result, the underlying prognostic mechanism of NLR and CRP might be different, at least in part. Therefore, in the present study, we evaluated the combination of ALI and CRP, and

Table 3. Multivariate Analysis

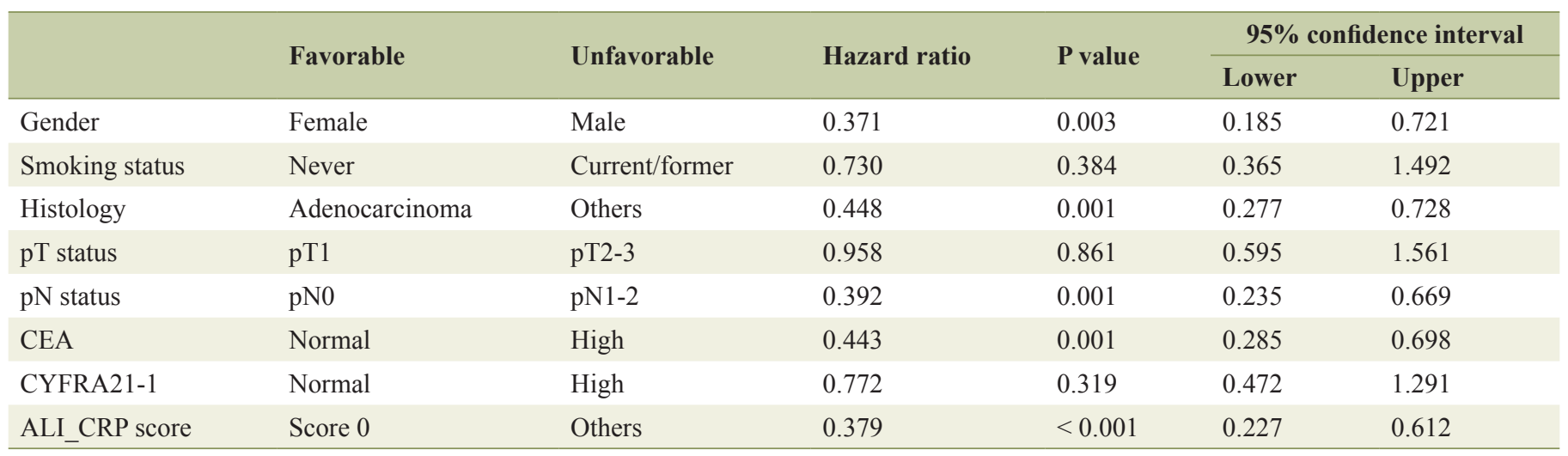

ALI: advanced lung cancer inflammation index; CRP: C-reactive protein; CEA: carcinoembryonic antigen; CYFRA21-1: cytokeratin 19 fragments. 
found the prognostic significance of the ALI CRP score. Our present result also showed that the ALI CRP score was related to gender, smoking status, histology, $\bar{p} \bar{S}$ tage, $\mathrm{pT}$ status, serum CYFRA21-1 level, but not related to age, $\mathrm{pN}$ status and serum CEA level. This indicated that the ALI_CRP score does not always correlate with a more aggressive disease phenotype. Furthermore, the results of multivariable analysis showed that the prognostic significance of the ALI_CRP score is independent.

The ALI CRP score has the advantage of being simple to measure, routinely available and well standardized. We believe that the ALI CRP is an inexpensive survival predictor of NSCLC patients following surgical resection and should be considered for routine clinical use.

The potential limitations of the current study included the use of a retrospective analysis and a small sample size from a single center. Thus, larger prospective studies will be needed to confirm these preliminary results.

\section{Conclusions}

The combination of ALI and CRP might serve as a potentially clinically valuable marker of patients' prognosis in operable NSCLC.

\section{Conflict of Interest}

The authors have declared that no conflict of interest exists.

\section{Author Contributions}

MT carried out most of the experiments and drafted the paper. TA and RM participated in the study design and completed the manuscript. MT conceived the study and carried out the statistical analysis. KN provided valuable insights for drafting the manuscript. All authors read and approved the final manuscript.

\section{References}

1. Balkwill F, Mantovani A. Inflammation and cancer: back to Virchow? Lancet. 2001;357(9255):539-545.

2. Mantovani A, Allavena P, Sica A, Balkwill F. Cancer- related inflammation. Nature. 2008;454(7203):436-444.

3. Roxburgh CS, McMillan DC. Role of systemic inflammatory response in predicting survival in patients with primary operable cancer. Future Oncol. 2010;6(1):149-163.

4. Leuzzi G, Galeone C, Gisabella M, Duranti L, Taverna F, Suatoni P, Morelli D, et al. Baseline C-reactive protein level predicts survival of early-stage lung cancer: evidence from a systematic review and meta-analysis. Tumori. 2016;102(5):441-449.

5. Liao C, Yu Z, Guo W, Liu Q, Wu Y, Li Y, Bai L. Prognostic value of circulating inflammatory factors in non-small cell lung cancer: a systematic review and meta-analysis. Cancer Biomark. 2014;14(6):469-481.

6. Tomita M, Ayabe T, Chosa E, Nakamura K. Prognostic significance of pre- and postoperative glasgow prognostic score for patients with non-small cell lung cancer. Anticancer Res. 2014;34(6):3137-3140.

7. Yin Y, Wang J, Wang X, Gu L, Pei H, Kuai S, Zhang Y, et al. Prognostic value of the neutrophil to lymphocyte ratio in lung cancer: A meta-analysis. Clinics (Sao Paulo). 2015;70(7):524-530.

8. Gu XB, Tian T, Tian XJ, Zhang XJ. Prognostic significance of neutrophil-to-lymphocyte ratio in non-small cell lung cancer: a meta-analysis. Sci Rep. 2015;5:12493.

9. Jafri SH, Shi R, Mills G. Advance lung cancer inflammation index (ALI) at diagnosis is a prognostic marker in patients with metastatic non-small cell lung cancer (NSCLC): a retrospective review. BMC Cancer. 2013;13:158.

10. Tomita M, Ayabe T, Nakamura K. The advanced lung cancer inflammation index is an independent prognostic factor after surgical resection in patients with non-smallcell lung cancer. Interact Cardiovasc Thorac Surg. 2017: ivx329.

11. Budczies J, Klauschen F, Sinn BV, Gyorffy B, Schmitt WD, Darb-Esfahani S, Denkert C. Cutoff Finder: a comprehensive and straightforward Web application enabling rapid biomarker cutoff optimization. PLoS One. 2012;7(12):e51862.

12. Tewari N, Martin-Ucar AE, Black E, Beggs L, Beggs FD, Duffy JP, Morgan WE. Nutritional status affects long term survival after lobectomy for lung cancer. Lung Cancer. 2007;57(3):389-394.

13. Jin Y, Zhao L, Peng F. Prognostic impact of serum albumin levels on the recurrence of stage I non-small cell lung cancer. Clinics (Sao Paulo). 2013;68(5):686-693. 